\title{
Multi-sensor data fusion for traffic planning and control
}

\author{
N. Corsi \& A. Capitanelli \\ D’Appolonia S.p.A., Italy
}

\begin{abstract}
Traffic and mobility are essential ingredients of modern society, as they are important prerequisites for economic and social prosperity. Due to opportunities provided by modern technologies, traffic management has become an important component in improving quality and safety, especially in road traffic. The objective of this paper is to present a novel approach to performing data correlation and to extend the developed techniques and processing strategies to satellite COSMO-SkyMed data $\left(\mathrm{CSK}^{\circledR}\right)$ in the framework of terrestrial Strategic Picture-based applications. By using an integrated system based on satellite, acoustic and GPS data, wide-area images of the entire road network can complement the selectively acquired data. The integration would take benefit of the enhanced imaging capabilities of COSMO-SkyMed and its high revisit rate given by the future constellation configuration, thus leading to fully exploitable in situ/EO integrated products. The preliminary test activity was performed in November 2010: there were promising results from the innovative system based on the heterogeneous data fusion, from Synthetic Aperture Radar to GPS and Acoustic Sensors.
\end{abstract}

Keywords: traffic management, vehicles detection, synthetic aperture radar.

\section{Introduction}

In the last years, a number of techniques have been tried to improve the management of traffic in city centres and on major roads but they have typically obtained limited success. A common concern is that the existing road network, by reason of being unmanaged, is inefficiently utilised and could better accommodate the traffic load placed on it. This would reduce the environmental impact by reducing the need for new roads and would reduce the time that road 
vehicles spend in traffic - often operating at their least efficient and most polluting. In addition, one should not be forget the improved economic efficiency that would result from a reduction in travel times and fuel consumption, as well as the reduction of accidents and the improvement in quality of life. The traffic-management techniques that have been tested have typically obtained limited success. The suggested reasons for this are as diverse as the techniques themselves, but one factor seems common amongst all the surveyed methods - the lack of adequate and accurate traffic information. It seems entirely logical to suggest that one cannot hope to manage a system without understanding the behaviour of that system and having accurate measurements of said behaviour.

Several methods have been developed to measure travel times or queue lengths at traffic signals (Neuman [1]), for example, because signalized intersections often are the crucial bottlenecks of urban road networks. However, non-signalized junctions might also be included as well as the estimation of further traffic state variables such as delay or travel time. However, all of them have their individual drawbacks. Currently, none of the existing detection methods seems to be able to reliably provide area-wide traffic condition information on its own. Furthermore, some methods have additional technical constraints. For example, video detection systems often have problems at night or during unfavourable weather conditions. Moreover, queue-length estimation at traffic signals based on loop detector data, for instance, typically has inherent limitations. This means that queues can only be measured within a certain range which mostly depends on the location of the loop detectors used. Because of all these problems, recent research activities focus on so-called data fusion methods which combine data from several data sources to enhance the quality and availability of traffic state information. Better algorithms should take advantage of the inherent correlations between data from several data sources directly.

Whatever the kind of application, it is important to gain detailed insights into the actual traffic dynamics. Without that, it is impossible to adequately describe the dependencies between relevant traffic state variables such as traffic demand, queue length and local density.

An important application in the field of traffic management might be the harmonization of traffic flow, i.e. the capability of keeping traffic flow stable for long periods in time and space. For example, high variations in speed and density could be suppressed by variable speed limits. Such a strategy is helpful for reducing lags, incidents, or fatalities, and keeps road traffic safe.

Traffic management is also essential for long-run data such as traffic engineering, planning, or operational activity. The collection of statistical traffic flow information is, in fact, essential for off-line planning and administration, computation of measures of effectiveness, and compilation of related statistical properties.

Generally, three basic parameters are utilised to characterise and manage the traffic information; they include the flow rate, the speed, and the density. Any of the following applications has its own requirements, according to these parameters. 
Surveillance and emergency response applications strongly rely on the knowledge of the traffic in the area of interest: numbers of vehicles, their position and kinematics, their routes and/or intentions, represent the necessary input for decision makers.

\subsection{Earth observation based systems}

In recent years, image processing technology has been widely deployed within both military and security surveillance systems.

Space based imaging has the characteristic of covering vast areas and has larger information quality as well as multi-spectral and repeat observations ability, thus, it is suitable for regional and global environment monitoring. The application of satellite images is not only used for mapping but also for monitoring temporal changes in the environment.

This technology has been used to provide essential, automated surveillance functions such as target detection, tracking and recognition using image data sourced in real-time from a variety of imaging sensors. Typical systems employing this technology include airborne and ground based surveillance systems.

The science of surveying and mapping encompasses a broad range of disciplines including Surveying and Mapping, Remote Sensing (RS), GIS and GPS.

EO provides two main sets of data of direct applicability to the traffic monitoring:

- Optical data;

- SAR (radar data).

Optical high resolution satellites are the main source of information to be used for land and infrastructure surveillance. They provide spectral information of the targets and areas monitored. This spectral information will serve the characterization of such targets.

The optical data presents two main constraints to be used for critical infrastructure monitoring: (i) Optical data is strongly influenced by weather conditions; (ii) Optical data is only provided in during the illuminated part of the orbit and in cloud-free scenes.

SAR satellites will constitute the other main source of information to enhance land and infrastructure security management and will also complement the use of optical imagery by overcoming some limitations, providing additional dimensions to the information and by bringing additional techniques of data analysis. SAR data have the characteristic of representing the object geometric features.

During the past years, increasing traffic appears to be one of the major problems in urban and sub-urban areas (Cambridge Systematics [2]). Optical systems are already in use (Reinartz et al. [3]) but are limited due to their daylight operation and cloud-free conditions requirements. (ii) Synthetic aperture radar (SAR) systems seem to be more promising due to their all-weather capability. 
With reference to the optical system, in fact, the primary factor influencing the possible service delivery frequency as well as the reliability of the service is the availability of satellite images. Many factors influence the satellite imaging frequency.

The primary factor influencing the satellite image delivery frequency is climatic conditions. A successful satellite image acquisition requires near perfect weather conditions, in particular the cloud coverage must be close to zero. Such conditions are not very common in the service case area.

Another central factor determining the satellite imaging frequency is the satellite revisit frequency. The satellites capable of producing images with a resolution sufficient for use in this project have a revisit time of many hours.

Synthetic aperture radar (SAR) sensors due to their all-weather capabilities seem to be well suited for such type of applications. Ground moving target indication (GMTI) approaches based on the displaced phase centre array (DPCA) technique are currently under investigation (Suchandt et al. [4]), but still suffer from low vehicle detection rate, sometimes below 10\%. Such low detection rates make it difficult to derive reliable information about the traffic flow parameters, e.g. vehicle density.

In the contest of the project, the dynamic aspect of traffic is a very important issue. It is clear that the dynamic aspect of traffic, especially in dense urban areas, is very important in understanding the build-up of traffic jams etc.

If satellite images taken with short time intervals could be made available then it might become possible to study the evolution of the traffic situation in an entire area, this could also possibly show how jams are formed etc.

Even though the number of images required for such a truly dynamic study might be difficult to obtain, it would be valuable if only a few images taken with some (short) time lapse between them could be made available. This could partly be used for analysing the dynamic aspect of different traffic situations, and partly it could be used for distinguishing between parked vehicles and vehicles that are in circulation.

EO-based technologies provide a valuable source of data for land and infrastructure monitoring by increasing the spatial and time coverage and by providing a routinely coherent source of data.

\section{System description}

The innovative system for traffic detection, control and planning, was developed within the SE-STP (Satellite Extended Strategic Traffic Picture) project that is a Research and Development activity funded by the Italian Space Agency (ASI) in the frame of the "Announcement of Opportunity" the prototype system was designed with a high level of scalability and modularity in order to be configured in optimum way depending on the dimensions, topography, vegetation, roads. It consists in (1) Heterogeneous Sensors Network (HSN); (2) Satellite Extended Module composed by the Earth Observation System (EOS); (3) Strategic Traffic Picture module (STP) composed by (3.a) Data Collector (DC), (3.b) Processing Unit (PU) and (3.c) the Visualization Console (VIS). 
The Heterogeneous Sensors Network (HSN) consists of GPS and in-situ Microphone Sensor (AA) devices and collects the position and the velocity information of the vehicles in the area where they are displaced.

The Satellite Extended Module (SE) is composed by the Earth Observation System (EOS) that is the logic unit undertaking the extraction of information concerning targets of interest from Cosmo-Skymed SAR images.

The Strategic Traffic Picture is the Module that contains Data Collector (DC), Processing Unit (PU) and the Visualization Console (VIS). The former is in charge of collecting the acquired data and storing them in dedicated database. Data can be accessed by the users through the Visualization Console (VIS). The DC is also able to transmit and synchronize the data flow in input to the Processing Unit (PU) for elaborating and extracting strategic information. This unit is in charge of SE-STP compilation where it will be possible to extract information related to each target (position, speed) and messages of intelligence to send to the operator.

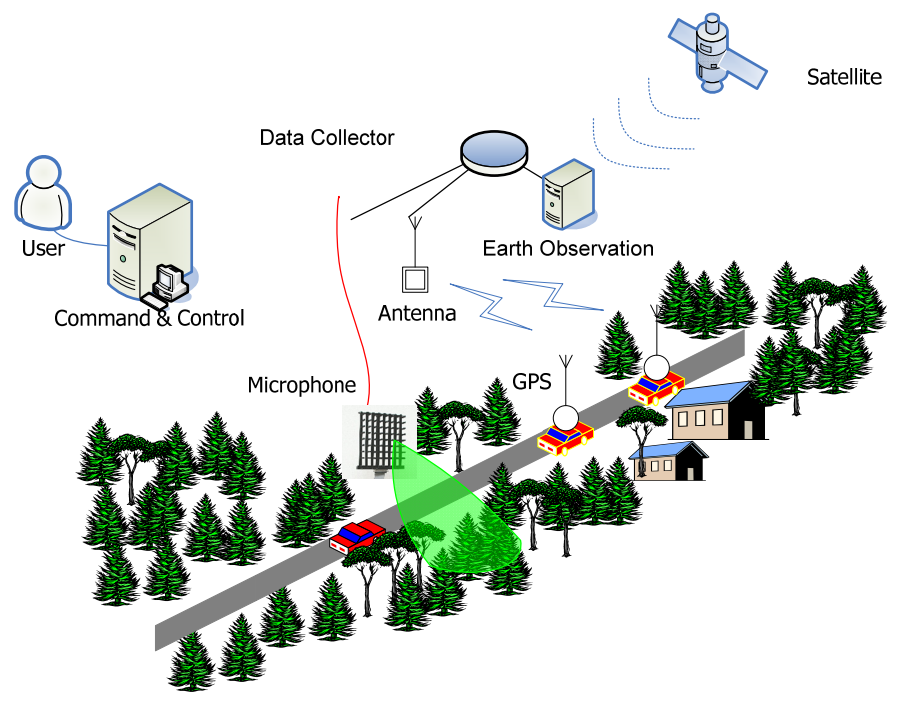

Figure 1: Typical scenario.

Acoustic sensors were used to detect locally and count vehicles; thanks to its accuracy the system was installed to continuously perform scanning in the monitored area, particularly focusing on vehicle distribution. Furthermore GPS devices were installed on cooperative vehicles in order to achieve an accurate traffic picture in the area of interest and to provide reference for other measures. GPS were easily integrated thanks to their autonomous power supply and the fact that they do not require maintenance.

Data provided by satellite overcome the limits introduced by terrestrial sensors about limited monitoring of area of interest. On the basis of the type of required image, the coverage of the area varies from 10 to $100 \mathrm{~km}$ of extension. 
Before providing the tests of integrated subsystems, it was necessary to receive the Satellite Image from the Italian Space Agency. This aspect is at basis of the validation of whole system: the image indeed determines the time synchronization for each subsystem. The final Strategic Traffic Picture aims at visualizing the actual scenario of vehicle distribution at a given instant of time.

The SAR image was taken at 18:04:54: the image was processed and analysed to extract the position of moving target.

\subsection{Characteristics of input data}

The input of the processing chain is a spotlight product characterized by the $1 \mathrm{~B}$ processing level. This is an amplitude image, ground-projected with a ground resolution of 1 meter $\times 1$ meter and characterized by 0.5 meters $\times 0.5$ meters a pixel spacing. For the technical discussion hereafter reported, the image related to an area located in Fiumesanto near the city of Sassari (Sardinia, Italy) will be used. The original image, provided by the ASI help desk, is shown. In particular the sea, the power plant with other industrial factories, some roads and a large area occupied by fields and vegetation in general are identified. Some speckles are present in textures and structures. In the next subsections the technical approach is described staring form the first statistic analysis to an accurate development method of the processing.
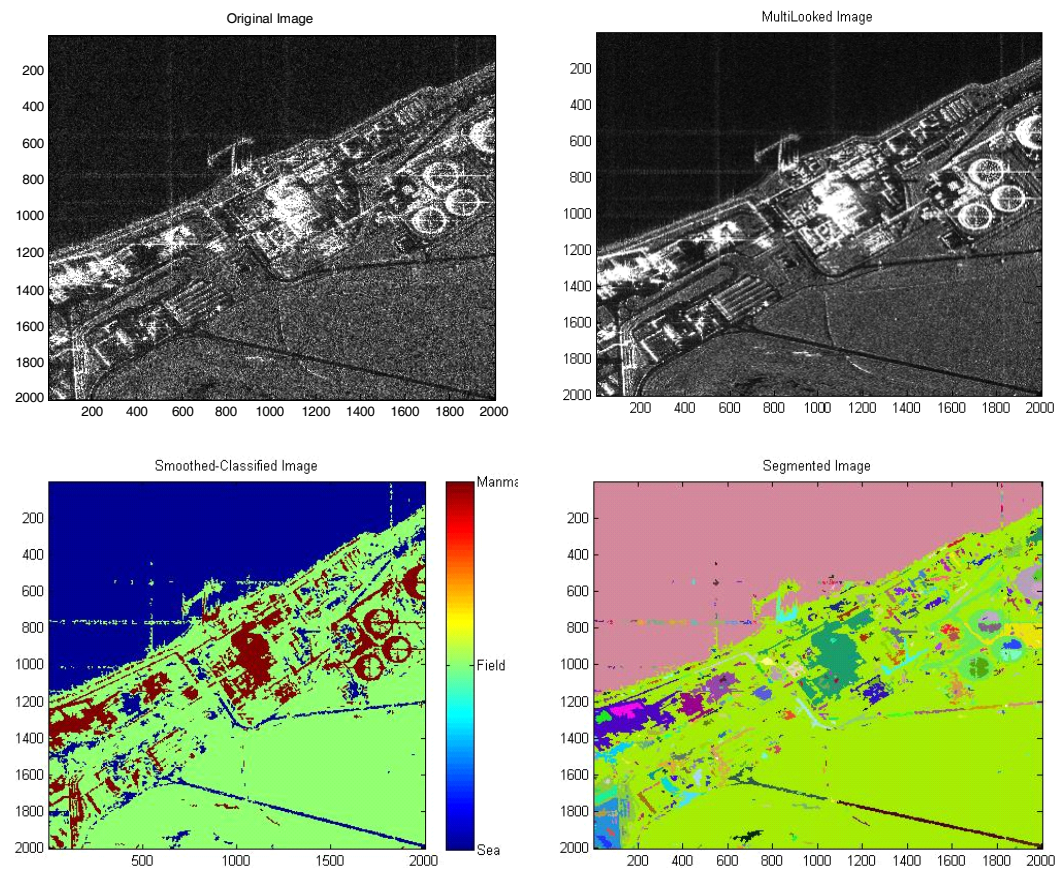

Figure 2: $\quad$ Image processing phases. 
The original image (Data provided by the Italian Space Agency) is filtered to reduce the background noise due to the scattering objects, false targets, lack of resolution. The second step consists in filtering of the image. The third phase of processing is to classify the image: the intent of the classification process is to categorize all pixels into one of several land cover classes. The objective of the image classification is to identify and portray the features occurring in an image in terms of the object or type of land cover these features actually represent on the ground. The next step is the segmentation process, the partition of an image into multiple segments in order to simplify the representation of the image into something that is more meaningful and easier to analyse. This process is typically used to locate the objects and boundaries in image and assigns a label to every pixel in an image such that pixels with the same label share certain visual characteristics.

Finally the system uses a database which contains GIS (Geographical Information System) information related to (i) the transportation infrastructures and to (ii) the buildings. Because the GIS information is stored in a vectorial structure, it must be loaded and projected onto the SAR image (which is, on the contrary, a raster image). In this way, the processor is able to detect (i) the roads, which represent the most likely place to find vehicles and the (ii) buildings, on which it is possible to exclude the presence of vehicles in transit.

However, since the SAR platform is in relative movement with terrain, the vehicles (and moving target in general) are focused with an azimuthal displacement (i.e., in along-track direction), if the target has a cross-track velocity component and deblurred, if the target has an along-track velocity component.
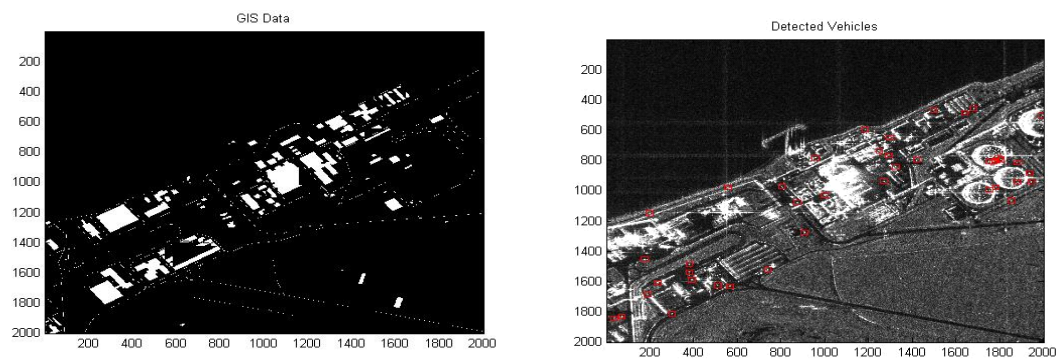

Figure 3: GIS integration and vehicles detection.

Therefore, the processor delimits an area for searching the target, around each road, which is calculated on the basis of the maximum velocity expected for the target and the angle of the road segment respect to the along-track direction of the satellite, according to the following relationship:

$$
\Delta A z=R_{0} \frac{v_{y} \sin \theta}{v_{s}}
$$

In the eqn (1), $\mathrm{R}_{0}$ is the slant-range distance of platform, $v_{\mathrm{y}}$ is the across track velocity of target, $v_{\mathrm{s}}$ is the velocity of satellite platform and $\theta$ is the angle between slant-range direction and the nadir of platform. 
The integration with GIS is an optional stage that can be chosen by the user during the image processing, in order to have a knowledge-based enhancement of target detection.

The last phase of the integer process is to apply the algorithms detection in order to extract the information on moving targets.

\subsection{Theoretical system performance}

During this developing phase of the project, two surveys were done in Fiumicino (Rome) which had environmental characteristics that made it a suitable place for system testing in terms of (i) good visibility of the roads, (ii) lack of background noise and (iii) low density of traffic. The goal of the first survey was to evaluate the performance of GPS and Acoustic Subsystem before integrating the whole system to the $\mathrm{CSK}^{\circledR}$ products. Both tests consist in recording the position data and the spectral signature coming from the GPS device and the acoustic system in order to track the vehicles and distinguish the cooperative from the non cooperative vehicle trough the Graphical User Interface. Once all data are recorded, the process step off-line data can begin: all data are fused together to provide a Strategic Traffic Picture monitored.

Before providing the tests of integrated subsystems, it was necessary to receive the Satellite Image from the Italian Space Agency. This aspect is at basis of the validation of whole system: the image indeed determines the time synchronization for each subsystem. The final Strategic Traffic Picture aims at visualizing the actual scenario of vehicle distribution at a given instant of time.

The SAR image was taken at 18:04:54: the image was processed and analysed to extract the position of moving target. The detection algorithm has highlighted the presence of some moving targets not only on the road but also in the area around the scenario. This is due the false alarms related to the scattering objects detected as targets. This is an current limitation of the algorithm that will be improved during the next months in the second part of the project.

From the picture is possible to see five true targets on the roads. The other alarms visible and highlighted through the red square could be moving targets displaced because of their speed.

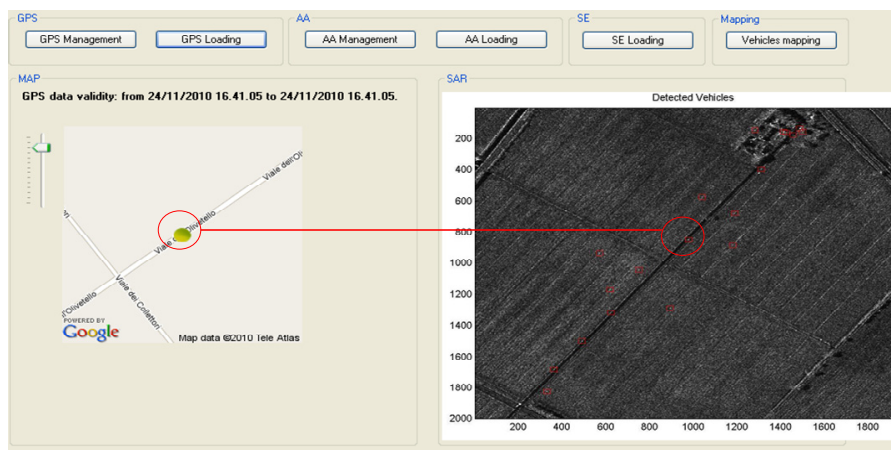

Figure 4: Acoustic and SAR data integration. 
The cooperative vehicle plotted on the left side of the map has the same position of the detected target at the same time (18:04:54): the validation on $\mathrm{CSK}^{\circledR}$ product is verified.

From the point of view of the acoustic system, the analysis of the scene is different. Indeed the acoustic system has not recorded any vehicle at that time exactly; before that instant of time and precisely at 18:04:10, 18:04:16, 18:04:30 and 18:04:44, the acoustic system recorded four vehicles.

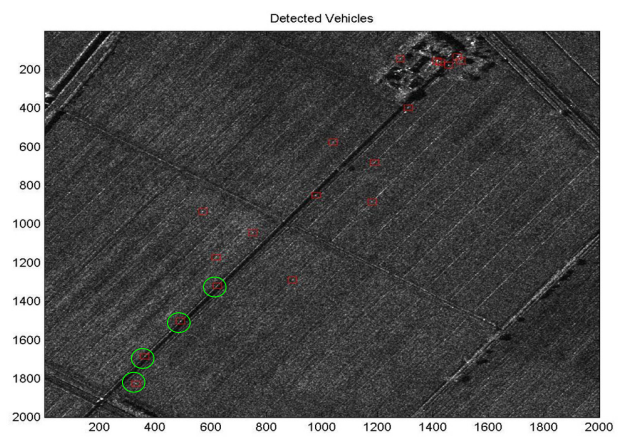

Figure 5: Non cooperative vehicles detected by acoustic system.

The complex scenario of terrestrial traffic picture is shown in Figure 6 as can be seen, the green icons related to the possible moving targets are plotted on the left side of the map with the acoustic sensor position (in violet) that indicates the presence of only one vehicle detected in a determined instant of time. On the right side of the interface, the SAR map is shown with all vehicles highlighted by the detection algorithm.

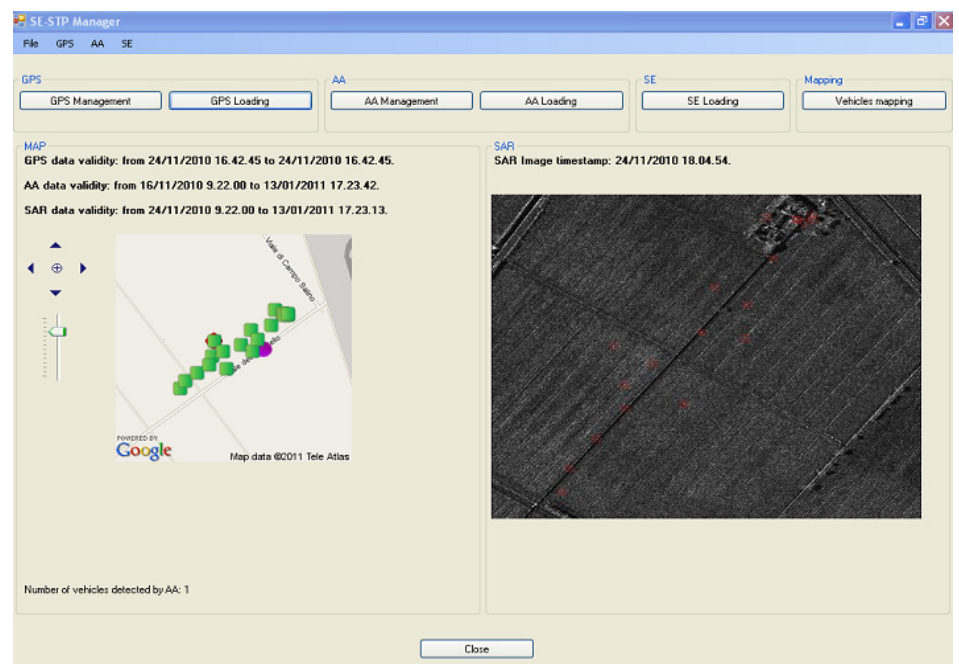

Figure 6: Completed scenario with all tracks and positions of moving targets. 
This information yields the Tactical Traffic Picture (TTP), which is exploited in monitoring applications in the security and safety domains: Traffic planning and control: (i) Traffic signalling planning and optimization (ii) Planning of transportation infrastructure (iii) Decision support for zoning regulations (iv) Maintenance of existing facilities and design of new facilities, (v) Safety analysis and (vi) Planning and allocation of resources in the event of emergences and disasters.

\section{Conclusions}

The SE-STP prototype System has been described showing the experimental results carried out in the first phase of the project and described showing the results of multi-technology integration and the benefits created in terms of traffic planning and control. Specifically, an innovative concept for Traffic Management has been introduced: it is based on Satellite data and SAR technology which is well suited to wide coverage and frequent monitoring of large areas.

The results reported in this paper clearly demonstrate the successful completion of the integration and pre-validation of the SE-STP demonstrator. Some specific problems have been encountered in this phase (i) the displacement of the moving targets due to SAR geometry and (ii) the lack of resolution of the satellite images. The detection of true alarms and the fusion of all data recorded and coming from heterogeneous sensors are the main aspects that make the development of an integrated terrestrial traffic monitoring system very challenging. These aspects will be developed to mitigate these risks during the second phase of the project.

\section{Acknowledgements}

The research activity was carried out within the ASI (Italian Space Agency) Funded Announcement of Opportunity framework, and demonstrated as activity of the "Application of COSMO-SkyMed data to Satellite Extended Strategic Traffic Picture based applications". The authors would like to thank the Transport Department of Sardinia for the support to the activity and the Command of Municipal Police of Fiumicino, for the permits granted during the test phases.

\section{References}

[1] Neuman, T., Association for European Transport and Contributors HighQuality Urban Traffic Monitoring-Theory and Evaluation of Promising Data Fusion Methodology, 2009.

[2] Cambridge Systematics, with Texas Transportation Institute, Traffic Congestion and Reliability: Trends and Advanced Strategies for Congestion Mitigation (http://ops.fhwa.dot.gov/congestion_report/), July 19, 2005. 
[3] Reinartz, P., Lachaise M., Schmeer E., Krauß T., and Runge H., Traffic Monitoring with Serial Images from Airborne Cameras, ISPRS Journal of Photogrammetry and Remote Sensing, vol. 61, pp. 149-158, 2006.

[4] Suchandt, S., Palubinskas G., Runge H., Eineder M., Meyer F., and Scheiber R., 2005a, An Airborne SAR Experiment for Ground Moving Target Identification, in Proc. ISPRS Hannover Workshop - High Resolution Earth Imaging for Geospatial Information, Hannover, vol. XXXVI, part I/W3, 2005 . 